\title{
QUATERNION HOMOLOGY OF BANACH SPACE
}

\author{
Y. GH. GOUDA AND H. N. ALAA
}

\begin{abstract}
In this article we are concerned with the quaternion Banach spaces and their homology. We obtain the relation between the quaternion and the dihedral homology for a unital involutive Banach algebra.
\end{abstract}

\section{Introduction}

It is well known that is there are two types of homological algebra theory; the homology theory of discrete algebras, initiated and developed by Hochschild 1945-1947 [7], [8], [9] and the homology theory of operator algebras studied by Jonson, Kadison and Ringrose [4], [5], [6]. In 1983 Connes and Tsygan [2], [14] have introduced a new type, the cyclic (co)homology of a unital algebra. The analog Banach cyclic (co)homology has been studied by Helemskii [10], Christensen and Sinclair [1] and Wodziski [15]. In 1987 Loday [13], and Krassawkas, Lapin and Solovev [12] introduced and studied the reflexive and dihedral (co)homology of involutive unital algebras. The author studied the analog reflexive and dihedral (co)homology of $C^{*}$-algebras [11]. The dihedral homology of algebras and its relation with quaternion homology has been studied by Loday [13]. In this article we are concerned with the quternion Banach spaces and their homology. Firstly we recall the definition and some properties of generalized quternion group.

\section{Generalized Quaternion Group $Q_{m}$}

Let $\mathbb{H}$ be algebra of quaternions $\mathbb{R} \oplus \mathbb{R}_{i} \oplus \mathbb{R}_{j} \oplus \mathbb{R}_{k}$. For every natural number $m \geq 2$, the generalized quaternion group $Q_{m}$ is defined as a subgroup of the multiplicative group $\mathbb{H}^{*}$, generated by the elements $x=e^{\pi i / m}$ and $y=j$. It is clear that the element $x$ has order $2 m$ and the relations $y^{2}=x^{m}$ and $y x y^{-1}=x^{-1}$ are fulfilled. Hence $x^{m} y^{-1}=y x y^{-1} y x y^{-1} \cdots y x y^{-1}=x^{-m}$ and we deduce that

$$
y \cdot y^{2} \cdot y^{-1}=y^{-2}, \quad \text { i.e. } \quad x^{2 m}=y^{4}=1 .
$$

Thus, the cyclic subgroup $C$ generated by the element $x$, is a normal subgroup and has index two in $Q_{m}$. It follows that the group $Q_{m}$ itself has order $4 m$. Let us list the most important properties of the generalized quaternion group $Q_{m}$ :

Received December 15, 1997.

1991 Mathematics Subject Classification. Primary 55N91; Secondary 55P91, 55Q91.

Key words and phrases. Dihedral homology, quaternion homology, Banach spaces, homology. 
(i) The group $Q_{m}$ is given by a co-representation

$$
Q_{m}=\left\{x, y ; x^{m}=y^{2}, y x y^{-1}=x^{-1}\right\} .
$$

(ii) In the extension

$$
0 \rightarrow C \rightarrow Q_{m} \rightarrow \mathbb{Z} / 2 \rightarrow 0
$$

the generator of $\mathbb{Z} / 2$ acts on $C$ as the multiplication by -1 .

(iii) Every element in the set $Q_{m} / C$ has order 4 .

(iv) An extension

$$
0 \rightarrow C \rightarrow Q_{m} \rightarrow \mathbb{Z} / 2 \rightarrow 0
$$

is not splittable.

Proposition 1.1. Let $\mathbb{R}$ be a commutative ring with unit. Then there exist 4 -periodic resolution of the trivial $Q_{m}$-module $\mathbb{R}$ :

$$
\cdots \rightarrow R\left[Q_{m}\right] \stackrel{N}{\longrightarrow} R\left[Q_{m}\right] \stackrel{w}{\longrightarrow} R\left[Q_{m}\right]^{2} \stackrel{v}{\longrightarrow} R\left[Q_{m}\right]^{2} \stackrel{u}{\longrightarrow} R\left[Q_{m}\right] \stackrel{\varepsilon}{\longrightarrow} R \longrightarrow 0 .
$$

where $\varepsilon$ is the natural augmentation,

$$
\begin{aligned}
& u=(1-x, 1-y), v=\left[\begin{array}{ll}
T & 1+x y \\
-(1+y) & x-1
\end{array}\right], w=\left[\begin{array}{l}
1-x \\
y x-1
\end{array}\right], T=1+x+x^{2}+\cdots+x^{m-1}, \\
& N=\sum_{g \in Q_{m}} g=\left(1+y^{2}+y^{3}+y\right) T .
\end{aligned}
$$

Proof. We use Fox's derivatives [1]. Let

$$
G=\left\{g_{1}, \ldots, g_{k} / r_{1}, \ldots, r_{\ell}\right\}
$$

be a group generated by the elements $g_{1}, \ldots, g_{k}$ with relations $r_{1}, \ldots, r_{\ell}$. The free differential $\partial r_{i} / \partial g_{j}$ of the group ring $\mathbb{Z}[G]$ is defined by:

$$
\frac{\partial(a b)}{\partial g}=\frac{\partial a}{\partial g}+a \frac{\partial b}{\partial g}, \quad \frac{\partial g}{\partial g}=1, \quad \frac{\partial h}{\partial g}=0,
$$

where $h$ is any generator of $G$ not equal to $g$. Then according to Fox [1], the sequence

$$
\mathbb{Z}[G]^{\ell} \stackrel{v}{\longrightarrow} \mathbb{Z}[G]^{k} \stackrel{u}{\longrightarrow} \mathbb{Z}[g]^{k} \stackrel{\varepsilon}{\longrightarrow} \mathbb{Z}, \longrightarrow 0,
$$

where $\varepsilon(g)=1, u=\left(1-g_{1}, \ldots, 1-g_{k}\right), v=\left(\frac{\partial r_{i}}{\partial g_{j}}\right), 1 \leq i \leq k, \leq j \leq \ell$ is the first part of the free resolution of the trivial G-module $\mathbb{Z}$.

By using the Fox's derivatives of the generalized quaternion group $Q_{m}$, when $k=2$, $\ell=2, g_{1}=x, g_{2}=y, r_{1}=x^{m} y^{-2}, r_{2}=x y x y^{-1}$ and $u=(1-x, 1-y)$,

$$
v=\left[\begin{array}{ll}
\frac{\partial\left(x^{m} y^{-2}\right)}{\partial x} & \frac{\partial\left(x y x y^{-1}\right)}{\partial x} \\
\frac{\partial\left(x^{m} y^{-2}\right)}{\partial y} & \frac{\partial\left(x y x y^{-1}\right)}{\partial y}
\end{array}\right]=\left[\begin{array}{ll}
1+x+\cdots+x^{m-1} & 1+x y \\
-(1+y) & x-1
\end{array}\right],
$$


We get the following exact sequence

$$
R\left[Q_{m}\right]^{2} \stackrel{v}{\longrightarrow} R\left[Q_{m}\right]^{2} \stackrel{u}{\longrightarrow} R\left[Q_{m}\right] \stackrel{\varepsilon}{\longrightarrow} R \longrightarrow 0 .
$$

Considering in (1.1), the factor $\mathcal{H} m_{R\left[Q_{m}\right]}\left(-, R\left[Q_{m}\right]\right)$ and modifying the $Q_{m}$-module structure by means of the isomorphism $f: Q_{m} \rightarrow Q_{m}, f(x)=x^{-1}, f(y)=(b y)^{-1}$ we get the exact sequence:

$$
\begin{aligned}
& \mathcal{H o m}_{R\left[Q_{m}\right]}\left(R\left[Q_{m}\right]^{2}, R\left[Q_{m}\right]\right) \stackrel{v^{*}}{\longleftarrow} \mathcal{H o m}_{R\left[Q_{m}\right]}\left(R\left[Q_{m}\right]^{2}, R\left[Q_{m}\right]\right) \stackrel{u^{*}}{\longleftarrow} \\
& \longleftarrow \mathcal{H o m}_{R\left[Q_{m}\right]}\left(R\left[Q_{m}\right], R\left[Q_{m}\right]\right) \stackrel{\varepsilon}{\longleftarrow} \mathcal{H o m}_{R\left[Q_{m}\right]}\left(R, R\left[Q_{m}\right]\right) \longleftarrow 0 .
\end{aligned}
$$

It is easy to verify that:

$$
\begin{aligned}
& u^{*}=(1-x, 1-y)=\left[\begin{array}{l}
1-x \\
y x-1
\end{array}\right]=w \\
& v^{*}=\left[\begin{array}{ll}
T & 1+x y \\
-(1+y) & x-1
\end{array}\right]^{*}=\left[\begin{array}{ll}
T & 1+x y \\
-(1+y) & x-1
\end{array}\right]=v \quad \text { and we get }
\end{aligned}
$$

the following exact sequence:

$$
0 \longrightarrow R \stackrel{\varepsilon^{*}}{\longrightarrow} R\left[Q_{m}\right] \stackrel{w}{\longrightarrow} R\left[Q_{m}\right]^{2} \stackrel{v}{\longrightarrow} R\left[Q_{m}\right]^{2} .
$$

Since the composition $\varepsilon^{*} \circ \varepsilon$ is a homomorphism $N$, from (1.1) and (1.2) we get the required 4-periodic resolution.

\section{Quaternion Bamach Spaces}

Let $E=\otimes_{n \geq 0} E_{n}$ be a graded Banach space over the field of complex numbers $\mathbb{C}$.

Consider the families of continuous linear maps on $E$ :

$$
\begin{gathered}
d_{n}^{1}: E_{n} \rightarrow E_{n-1}, \quad s_{n}^{j}: E_{n} \rightarrow E_{n+1^{\prime}} \\
\tau_{n}, \omega_{n}: E_{n} \rightarrow E_{n}, \quad 0 \leq i \leq n, 0 \leq j \leq n,
\end{gathered}
$$

which satisfy the following conditions

$$
\begin{aligned}
d_{n}^{i} d_{n+1}^{j} & =d_{n}^{j-1} d_{n+1}^{i}, \quad i<j, \\
s_{n+1}^{i} s_{n}^{j} & =s_{n+1}^{j+1} s_{n}^{i}, \quad i \leq j, \\
d_{n}^{i} s_{n-1}^{j} & = \begin{cases}s_{n-2}^{j-1} s_{n-1}^{j}, & i<j, \\
I d\left(E_{n-1}\right), i=j, \quad j+1, & \\
s_{n-2}^{j} d_{n-1}^{i-1}, \quad i>j,\end{cases} \\
d_{n}^{i} \tau_{n} & =\tau_{n-1} d_{n}^{i-1}, \quad s_{n}^{i} \tau_{n}=\tau_{n+1} s_{n}^{i+1}, \quad 1 \leq i \leq n, \\
d_{n}^{j} \omega_{n} & =\omega_{n-1} d_{n}^{n-j}, \quad s_{n}^{j} \omega_{n}=\omega_{n+1} s_{n}^{n-j}, \quad 0 \leq j \leq n, \\
\tau_{n}^{n} & =\omega_{n}^{2}, \omega_{n} \tau_{n} \omega_{n}^{-1}=\tau_{n}^{-1} .
\end{aligned}
$$


A graded Banach space $E=\oplus_{n \geq 0} E_{n}$ considered together with these families of continuous linear maps is called a quaternion Banach space. An arbitrary unital Banach algerbra $A$ gernerates the quaternion Banach space.

Indeed, put

$$
E_{n}=A \hat{\otimes} A \hat{\otimes} A \cdots \hat{\otimes} A(n+1 \text { times }),
$$

where $\hat{\otimes}$ is the continuous tensor product in the sense of Grothendiec. Define operators:

$$
d_{n}^{i}: E_{n} \rightarrow E_{n-1}, \quad s_{n}^{j}: E_{n} \rightarrow E_{n+1}
$$

by means of the formulas

$$
\begin{aligned}
& d_{n}^{i}\left(a_{0} \otimes, \ldots \otimes a_{n}\right)=a_{0} \otimes \cdots a_{i} a_{i+1} \otimes \cdots \otimes a_{n}, \quad 0 \leq i<n, \\
& d_{n}^{n}\left(a_{0} \otimes, \ldots \otimes a_{n}\right)=a_{n} a_{0} \otimes \cdots \otimes a_{n-1}, \\
& s_{n}^{j}\left(a_{0} \otimes, \ldots \otimes a_{n}\right)=a_{0} \otimes \cdots \otimes a_{j} \otimes e \otimes a_{j+1} \otimes \cdots \otimes a_{n}, \quad 0 \leq j<n, \\
& s_{n}^{n}\left(a_{0} \otimes, \ldots \otimes a_{n}\right)=e \otimes a_{0} \otimes \cdots \otimes a_{n} .
\end{aligned}
$$

Moreover, define the operators $\tau_{n}: E_{n} \rightarrow E_{n}, \omega_{n}: E_{n} \rightarrow E_{n}$, putting

$$
\begin{aligned}
\tau_{n}\left(a_{0} \otimes, \ldots \otimes a_{n}\right) & =(-1)^{n} a_{n} \otimes a_{0} \otimes \ldots \otimes a_{n-1} \\
\omega_{n}\left(a_{0} \otimes, \ldots \otimes a_{n}\right) & =\alpha(-1)^{\frac{n(n+1)}{2}} a_{0}^{*} \otimes a_{n}^{*} \otimes \cdots \otimes a_{1}^{*},
\end{aligned}
$$

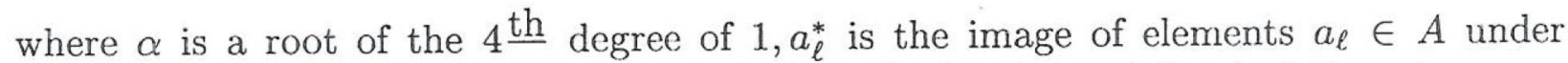
involution $*: A \rightarrow A$. It is easy to verify that the family so defined of Banach spaces and continuous linear maps is a quternion Banach space. In what follows we denote the quaternion Banach space by:

$$
Q(A): Q(A)_{n}=A \hat{\otimes} \cdots \hat{\otimes} A(n+1 \text { - times }) .
$$

\section{Continuous Quaternion Homology}

Proposition 3.1. Let $E=\oplus_{n \geq 0} E_{n}$ be a quaternion Banach space. Put

$$
t_{n}=(-1)^{n} \tau_{n}, r_{n}=(-1)^{\frac{n(n+1)}{2}} \alpha \omega_{n},
$$

where $\alpha=1,-1, i,-i$. Then there exists a bicomplex ${ }^{\alpha} \mathcal{E}(E)$ with 4-periodic rows:

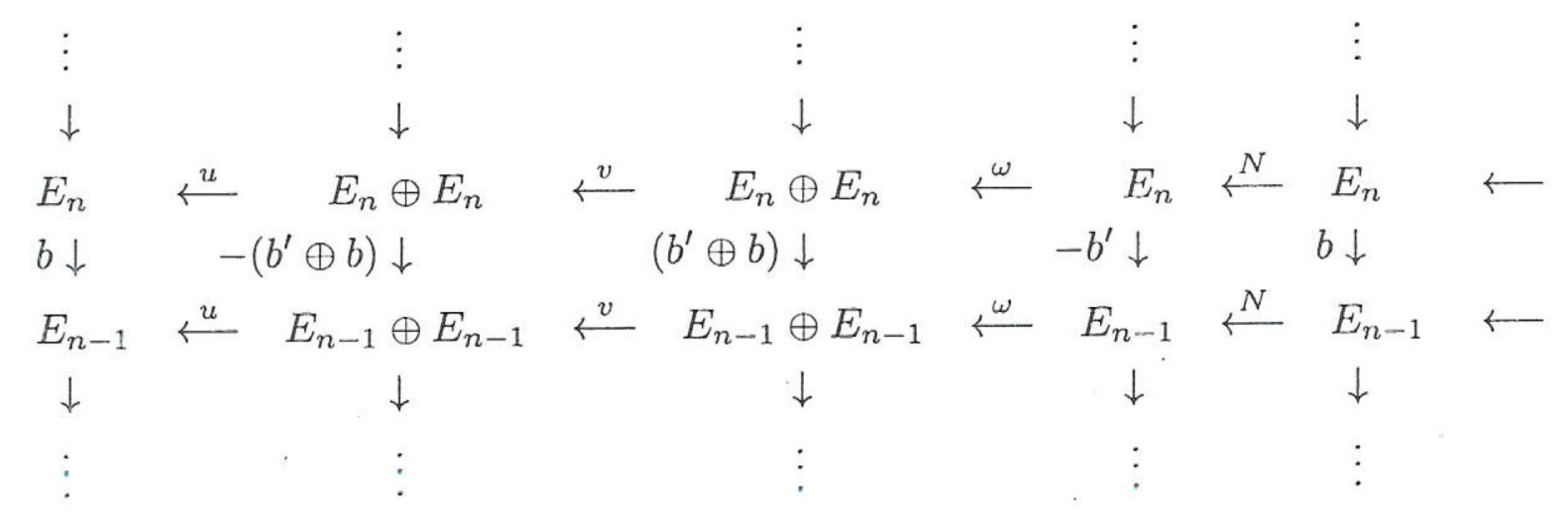


where $u=(1-t, 1-r), v=\left[\begin{array}{ll}T & 1-t r \\ -1+r & t-1\end{array}\right], w=\left[\begin{array}{l}1-t \\ -r t-1\end{array}\right], T=1+t+\cdots+t^{n-1}$, $N=\left(1+r+r^{2}+r^{3}\right) T, b=\sum_{i=0}^{n}(-1)^{i} d^{i}, b^{\prime}=\sum_{i=0}^{n-1}(-1)^{i} d^{i}$.

Proof. This assertion follows immediately from the following:

$$
b(1-t)=(1-t) b^{\prime}, \quad b r=r b, \quad b^{\prime} d r=d r b^{\prime}, \quad b^{\prime} T=T b, \quad b^{\prime} N=N b .
$$

Definition 3.2. [3]. Let $E=\oplus_{n \geq 0} E_{n}$ be a quaternion Banach space. Define the quaternion homology of $E$ by the formula:

$$
{ }^{\alpha} \mathcal{H} Q_{n}(E)=\mathcal{H}_{n}\left(\operatorname{Tot}^{\alpha} \mathcal{E}(E)\right)
$$

Let $E=Q_{n}(A)$, then

$$
{ }^{\alpha} \mathcal{H} Q_{n}(E)=\mathcal{H}_{n}\left(\operatorname{Tot}^{\alpha} \mathcal{E}(A)\right)
$$

Consider now the bicomplex consisting the first four columns of the bicomplex ${ }^{\alpha} \mathcal{E}(E)$, we shall denote it by ${ }^{\alpha} P(E)$, and suppose the following exact sequence

$$
\left.0 \longrightarrow^{\alpha} P(E) \longrightarrow \operatorname{Tot}^{\alpha} \mathcal{E}(E A) \stackrel{q}{\longrightarrow} \operatorname{Tot}^{\alpha} \mathcal{E}(A)\right) \longrightarrow 0
$$

Following [13] the homology of the complex ${ }^{\alpha} P(E)$ is a periodic and given by:

$$
{ }^{\alpha} \mathcal{H} P_{n}(E)=\mathcal{H}_{n}\left(\operatorname{Tot}^{\alpha} P(E)\right) \text {. }
$$

Since the bicomplex ${ }^{\alpha} \mathcal{E}(E)$ has 4 -periodic rows, we get the following exact sequence relating the periodic homology with quternion homology.

Theorem 3.3. There exists the exact sequence

$$
\begin{aligned}
& \longrightarrow^{\alpha} \mathcal{H} P_{n}(E) \longrightarrow^{\alpha} \mathcal{H} Q_{n}(E) \longrightarrow^{\alpha} \mathcal{H} Q_{n-4}(E) \longrightarrow \\
& \longrightarrow^{\alpha} \mathcal{H} P_{n-1}(E) \longrightarrow^{\alpha} \mathcal{H} Q_{n-1}(E) \longrightarrow^{\alpha} \mathcal{H} Q_{n-5}(E) \longrightarrow
\end{aligned}
$$

Following [12], the relation between ${ }^{\alpha} \mathcal{H} P_{n}(E)$ and the dihedral homology is given by:

$$
\begin{aligned}
& \longrightarrow^{\alpha} \mathcal{H} P_{n}(E) \longrightarrow^{\alpha} \mathcal{H} D_{n}(E) \longrightarrow^{\alpha} \mathcal{H} D_{n-4}(E) \longrightarrow \\
& \longrightarrow^{\alpha} \mathcal{H} P_{n-1}(E) \longrightarrow^{\alpha} \mathcal{H} D_{n-1}(E) \longrightarrow^{\alpha} \mathcal{H} D_{n-5}(E) \longrightarrow
\end{aligned}
$$

Comparing the relations (3.1) and (3.2) we get the relation between dihedral and quternion homology in the following.

Proposition 3.4. There exist the following natural isomorphism:

$$
\begin{array}{r}
{ }^{1} \mathcal{H} Q_{n}(A) \cong{ }^{1} \mathcal{H} d_{n}(A), \\
{ }^{-1} \mathcal{H} Q_{n}(A) \cong{ }^{-1} \mathcal{H} D_{n}(A),
\end{array}
$$

Similarly, one can define a quaternion cohomology for an unital Banach algebra with an involution and get the results. 


\section{References}

[1] E. Christensen and A. M. Sinclair, On the vanishing of $H^{n}\left(A, A^{*}\right)$ for certain $c^{*}$-algebras, Pasific J. of Math. 137(1989), 55-63.

[2] A. Connes, Cohomologie cyclique et functeur EXT ${ }^{n}$, C. R. Acad. Sci. Paris. Ser. A., 296(1983), 953-958.

[3] R. M. Fox, Free differential calculus, Ann. Math., 57 (1953), 547-560.

[4] B. E. Johnson, Cohomology of operator algebras, Memoirs Amer. Math. Soc. (1972).

[5] B. E. Johnson, J. R. Kadison and J. R. Ringrose, Cohomology of operator algebras I. Type I von Neumann algebras, Acta Math. 126 (1971), 227-243.

[6] B. E. Johnson, J. R. Kadison and J. R. Ringrose, Cohomology of operator algebras III. Reduction to normal cohomology, Bull. Soc. Math. France 100(1972), 73-96.

[7] G. Hochschild, On the cohomology groups of an associative algebra, Ann. of Math. 46(1945), 58-67.

[8] - - On the cohomology theory for associative algebra, Ann. of Math. 47(1946), 568-579.

[9] _- Cohomology and representaions of associative algebra, Duke Math. J. 14(1947), 921948.

[10] - Banach cyclic (co)homology as Banach derived function, St. Petersburg Math. J. 3(1992), 1149-1164.

[11] Y. Gh. Gouda, The homology with inner symmetry of $C^{*}$-algebra, Ph. D. thesis, Moscow State University, 1993.

[12] R. L. Kpasauskas, S. V. Lapen and Yu. P. Solovev, Dihedral homology and cohomology, Basic conception and constructions, Math. Sbornik. 133 (1987), 25-48.

[13] J-L. Loday, Homologie dihedrale et quaternionique, Advances in Math. 66 (1987), 119-148.

[14] B. Tsygan, The lie algebra of matrices and Hochschild homology, YMH. 38 (1983), 217-218 (in Russian).

[15] M. Wodzicki, Vanishing of cyclic homology of stable $C^{*}$-algebras, C. R. Acad. Sci. Paris 307 (1988), 329-334.

Department of Math., Faculty of Science, South Valley Univ., Aswan, Egypt. 